\title{
Magnetic and electric response of single subwavelength holes
}

\author{
N. Rotenberg,,${ }^{1, *}$ T. L. Krijger,,${ }^{1, \dagger}$ B. le Feber, ${ }^{1}$ M. Spasenović, ${ }^{1, \dagger}$ F. Javier García de Abajo, ${ }^{2,3}$ and L. Kuipers ${ }^{1}$ \\ ${ }^{1}$ Center for Nanophotonics, FOM Institute AMOLF, Science Park 104, 1098 XG, Amsterdam, The Netherlands \\ ${ }^{2}$ ICFO-Institut de Ciencies Fotoniques, Mediterranean Technology Park, 08860 Castelldefels (Barcelona), Spain \\ ${ }^{3}$ ICREA-Institucio Catalana de Recerca i Estudis Avançats, Passeig Lluís Companys, 23, 08010 Barcelona, Spain
}

(Received 25 October 2013; published 20 December 2013)

\begin{abstract}
We use polarization-resolved near-field measurements, in conjunction with electromagnetic theory, to separate and quantify the electric and magnetic optical response of subwavelength holes in thick gold films. Using $1550 \mathrm{~nm}$ light, we determine the amplitudes of the electric and magnetic polarizabilities of holes with diameters ranging from 600 to $1000 \mathrm{~nm}$. Additionally, we study the scattered field distributions that arise from the interactions of the holes with surface plasmon polaritons, and show that forward-backward scattering ratios as high as $2.5: 1$ are possible. Our study provides experimental access and theoretical understanding of the full electromagnetic polarizability that describes the optical response of metallic holes at telecom wavelengths, which is a prototypical structure in currently explored optical signal processing and sensing devices.
\end{abstract}

PACS number(s): 73.20.Mf, 78.68.+m, 73.50.Bk

Nanoscopic structures of small size compared to the wavelength of light can display both an electric and a magnetic response. ${ }^{1}$ This is true even in the case where the constituent materials of the structure all have a near unity magnetic permeability. The geometry of the nanostructure confers a magnetic response and is, in fact, the underlying principle of many metamaterials. ${ }^{2,3}$ This electromagnetic response, which in metamaterials can lead to a negative refractive index and hence cloaking or superresolution imaging, also plays a role in a variety of plasmonic phenomena ranging from extraordinary optical transmission ${ }^{4-6}$ to the confinement and control of light at the nanoscale. ${ }^{7-9}$

Surprisingly, outside of the field of metamaterials, ${ }^{10}$ the magnetic response of nanoscopic structures has traditionally been neglected. Happily, this trend is changing, and recently researchers have developed various techniques to map the optical magnetic field, ${ }^{11-13}$ and have quantified the emission of light from magnetic dipoles. ${ }^{14}$ Further, there have been several recent investigations into Mie scattering of dielectric spheres, where the interference between the magnetic and electric responses results in directional emission. ${ }^{15-17}$ Likewise, interest in magnetoplasmonic nanostructures is rapidly growing, both for those that have magnetic constituents ${ }^{18}$ and for those that do not. ${ }^{19}$

An exemplary nanoplasmonic structure which has recently received considerable attention is a dielectric-filled hole in a metallic film, ${ }^{6,20-24}$ due in part to its aforementioned connection to both extraordinary optical transmission and metamaterials. However, even for this relatively simple structure there have been only a few hints at the presence of a magnetic optical response, ${ }^{23,24}$ although its magnitude is expected to be comparable to the electric response. ${ }^{6}$ A direct observation of both the electric and the magnetic responses of such types of nanoscale objects is still missing, and in particular an experimental quantification of their strengths.

Here we address this important aspect of the response of metallic holes, using a series of phase- and polarizationsensitive near-field optical measurements to determine the amplitude of both the electric and magnetic polarizabilities of subwavelength holes in a gold film. We show that we are able to describe the nanoscopic light-matter interaction in terms of an in-plane magnetic dipole and an out-of-plane electric dipole. By comparing our measurements with electromagnetic theory we are able to disentangle the surface wave radiated by each dipole. This allows us to show, for a plasmonic nanostructure, that the asymmetry and high directionality of the scattered field is due to the interference of the magnetic and electric contributions.

To quantify the optical response of subwavelength holes we use the sample shown in Fig. 1, which consists of a surface plasmon polariton (SPP) launcher (slit and grating) and a single subwavelength hole perforated in a 200-nm-thick gold film supported on a glass substrate. The slit is illuminated from the glass side using a $1550 \mathrm{~nm}$ continuous laser source, launching SPPs that the grating directs efficiently towards the hole. ${ }^{25}$ In our coordinate system, which is shown in this figure, the SPPs propagate in the $x$ direction, towards the hole. The transverse, in-plane direction is $y$. We define the angle between any inplane vector and the $x$ axis to be $\varphi$.

The ensuing scattering event, which occurs when the SPP wave interacts with the hole, is imaged by a home-built nearfield microscope ${ }^{26}$ By placing the probe of the microscope at a height of $20 \mathrm{~nm}$ above the film, we are capable of converting the near fields into far-field radiation, which we then detect. Furthermore, because we use a heterodyne detection scheme, we measure both the amplitude and the phase of the outcoupled light, which are proportional to the amplitude and phase of the near field. Similarly, we can also resolve the in-plane orientation of the near field. That is, we are able to separately and simultaneously map the near fields that are oriented along $\hat{x}$ and $\hat{y}$.

Typical examples of the measured field maps for a $630 \mathrm{~nm}$ hole are shown in Figs. 2(a) and 2(b), for $x$ - and $y$-oriented which is located near fields, respectively. In the presence of the hole, near $(-3,-4) \mu \mathrm{m}$, we observe a marked increase in the field amplitude. This seems to indicate the presence of quasicylindrical waves ${ }^{22,27}$ which characteristically decay rapidly with distance away from the hole. Because the fields near the hole have both large amplitudes and gradients it is unclear how they are detected by the probe, and henceforth this region (within $500 \mathrm{~nm}$ of the hole) will be omitted from the analysis. Rather, our analysis will be based upon fields 


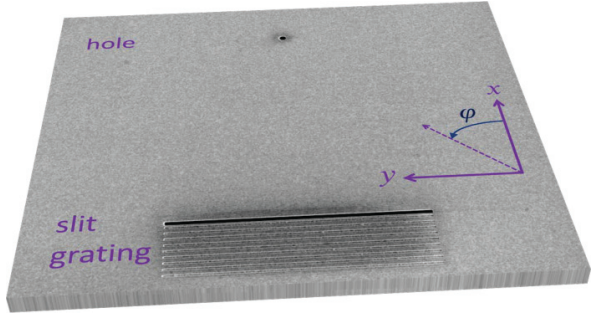

FIG. 1. (Color online) Scanning electron micrograph of the sample. The SPP propagates from the slit along the positive $x$ direction towards the subwavelength hole, as shown by the coordinate axes. The slit is $25 \mu \mathrm{m}$ in length and is carved $30 \mu \mathrm{m}$ away from the hole.

evolving in flat metal regions, for which we can assume a uniform interaction between tip and metal surface.

Although the field distributions seen in these images are far from trivial, we can immediately identify several distinct features that they share. First, in both images we see a beamlike feature centered about $y=0$, although in Fig. 2(a) it appears as a single, bright strip, while in Fig. 2(b) it becomes a double strip. Additionally, in both images we see parabolic fringes whose periodicity suggests that they arise due to interference.

In fact, the features that we observe in these images can be understood in terms of the incident and scattered waves associated with the hole-SPP interaction. Not surprisingly, the beamlike feature observed in both images is the incident SPPs. It is tempting to expect this feature only for the longitudinal fields [Fig. 2(a)], due to the nature of the SPPs. However, because we have an incident Gaussian SPP beam, and not a plane wave, we also observe a smaller amplitude, beamlike feature with transverse fields [Fig. 2(b)], which changes sign at the beam center, thus producing the observed double strip structure. The fringes that we observe can then be understood to arise from the interference of the incident SPP wave and the wave scattered by the hole.

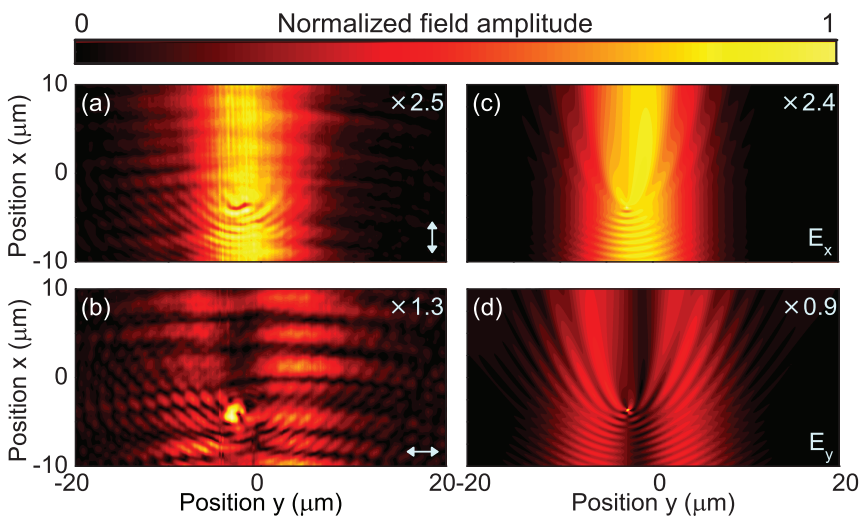

FIG. 2. (Color online) Polarization-resolved near-field maps of a SPP beam scattering from a $630 \mathrm{~nm}$ hole. Experimental measurements [(a), (b)] and theoretical calculations [(c), (d)] are shown for both $x$ and $y$ electric field orientations, as denoted at the bottom right of each image. The factors at the top right corners denote the amplitude relative to the color scale. Parabolic fringes due to the interference between the incident SPP and the scattered wave are clearly visible in all figures.
Intuitively, the scattering of SPPs from subwavelength holes, as shown in Fig. 2, can be understood as a three-step process: ${ }^{23}$ (1) An incident SPP beam propagates towards a hole. (2) At the location of the hole, the interaction of the SPP fields with the free electrons in the metal boundary of the hole induces dipoles. (3) These dipoles then radiate, and it is mainly the interference of the incident beam with those components that are scattered to surface plasmons that we actually detect. The second step is of particular interest, since it encapsulates the interaction of the electromagnetic field with the nanoscopic structure, our hole. However, as we show below, to quantify this interaction we must first accurately model both the incident $\left(\mathbf{E}^{\text {in }}, \mathbf{H}^{\text {in }}\right)$ and scattered $\left(\mathbf{E}^{\mathrm{s}}, \mathbf{H}^{\mathrm{s}}\right)$ fields that correspond to steps (1) and (3).

The three components of the incident Gaussian SPP beam above the film can be written as a Fourier sum of plane waves

$$
\begin{aligned}
& E_{x}^{\text {in }}(x, y, z)=-C \frac{w_{\mathrm{sp}}}{k_{0} \kappa_{\mathrm{sp}}} e^{i w_{\mathrm{sp}} z} \sum_{\kappa_{y}, \kappa_{x}} \kappa_{x} e^{-\left(\alpha^{2} / 2\right) \kappa_{y}^{2}} e^{i\left(\kappa_{x} x+\kappa_{y} y\right)}, \\
& E_{y}^{\mathrm{in}}(x, y, z)=-C \frac{w_{\mathrm{sp}}}{k_{0} \kappa_{\mathrm{sp}}} e^{i w_{\mathrm{sp}} z} \sum_{\kappa_{y}, \kappa_{x}} \kappa_{y} e^{-\left(\alpha^{2} / 2\right) \kappa_{y}^{2}} e^{i\left(\kappa_{x} x+\kappa_{y} y\right)}, \\
& E_{z}^{\mathrm{in}}(x, y, z)=C \frac{\kappa_{\mathrm{sp}}}{k_{0}} e^{i w_{\mathrm{sp}} z} \sum_{\kappa_{y}, \kappa_{x}} e^{-\left(\alpha^{2} / 2\right) \kappa_{y}^{2}} e^{i\left(\kappa_{x} x+\kappa_{y} y\right)},
\end{aligned}
$$

where the limits of summation, which reflect the explicit separation of the in-plane wave vector into its components, are $\kappa_{y} \in\left[-k_{0}, k_{0}\right]$ and $\kappa_{\mathrm{sp}}^{2}=\kappa_{x}^{2}+\kappa_{y}^{2}$. In these equations, $k_{0}=$ $2 \pi / \lambda$ is the wave vector of the incident light of wavelength $\lambda$, and $\kappa_{\mathrm{sp}}=k_{0} \sqrt{(\epsilon+1) / \epsilon}$ and $w_{\mathrm{sp}}=-k_{0} / \sqrt{\epsilon+1}$ are the in-plane and out-of-plane SPP wave vectors, respectively, where the complex dielectric function of gold at $1550 \mathrm{~nm}$ is $\epsilon=-115+11 i{ }^{28}$ Finally, $C$ and $\alpha$ determine the amplitude and width of the incident SPP beam. The corresponding magnetic field $\mathbf{H}^{\text {in }}$ is calculated from Eq. (1) using Ampere's law.

Likewise, we can write an analytic expression for the SPPs radiated by the hole dipoles. As shown in earlier work, ${ }^{23}$ for plasmonic scattering this radiation is dominated by an out-ofplane electric dipole $p_{z}$ and an in-plane magnetic dipole $m_{y}$, and hence it can be written as

$$
\begin{aligned}
\mathbf{E}^{\mathrm{s}}= & -2 \pi i \rho^{O} e^{i w_{\mathrm{sp} z}}\left[k_{0}^{2} \kappa_{\mathrm{sp}} H_{1}^{(1)}\left(\kappa_{\mathrm{sp}} r\right) \cos \varphi m_{y}\right. \\
& \left.+i k_{0} \kappa_{\mathrm{sp}}^{2} H_{0}^{(1)}\left(\kappa_{\mathrm{sp}} r\right) p_{z}\right]\left(\hat{\mathbf{r}}-\frac{\kappa_{\mathrm{sp}}}{w_{\mathrm{sp}}} \hat{\mathbf{z}}\right),
\end{aligned}
$$

where

$$
\rho^{O}=\frac{\epsilon}{(1+\epsilon)^{1.5}(1-\epsilon)} .
$$

In this equation, $H_{m}^{(1)}$ are Hankel functions, $r=$ $\sqrt{\left(x-x_{0}\right)^{2}+\left(y-y_{0}\right)^{2}}$ is the displacement from the hole position at $\left(x_{0}, y_{0}\right)$, and $\hat{\mathbf{r}}=(\cos \varphi \hat{\mathbf{x}}, \sin \varphi \hat{\mathbf{y}})$. A more general expression for the way dipoles radiate SPPs, which includes all of the different electric and magnetic dipole orientations, can be found in Ref. 23.

The total field of the scattering event, which includes contributions from both the incident and scattered SPPs, can then be written as $\mathbf{E}^{\text {in }}+\mathbf{E}^{\mathrm{s}}$. Using Eqs. (1) and (2), with $C, \alpha,\left(x_{0}, y_{0}\right)$, and the dipole strengths $p_{z}$ and $m_{y}$ as fitting 

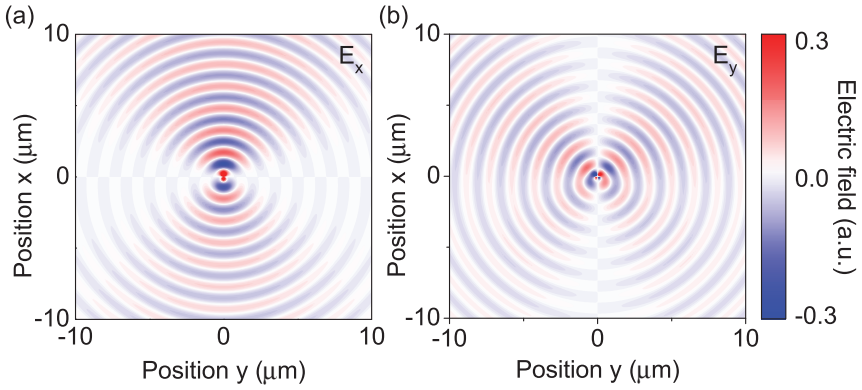

(c)

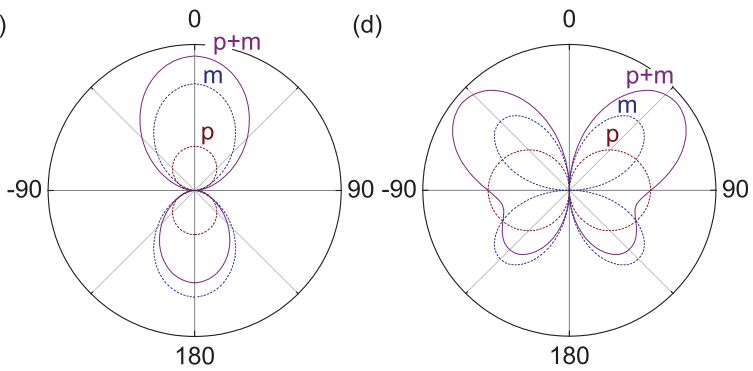

FIG. 3. (Color online) Calculated scattered SPP field for a $630 \mathrm{~nm}$ hole, with parameters as extracted from a fit to the measurement shown in Fig. 2. Both the (a) $x$ - and (b) $y$-oriented electric field components are plotted. (c),(d) Corresponding angular profiles of the field amplitudes (solid curves), as well as separate electric and magnetic contributions (dashed curves), which are labeled $p$ and $m$, respectively.

parameters to the experimental data for the 630-nm-diameter hole, produces the results shown in Figs. 2(c) and 2(d). This theoretical modeling is in good agreement with the measurements [Figs. 2(a) and 2(b)]. The uncertainty in the exact position of the hole, as it turns out, provides the main source of error for the fits. This uncertainty arises because the position of the interference fringes in our calculations is determined both by the position of the hole and the relative phases of the electric and magnetic dipoles. As we have no independent determination of the hole position, this uncertainty, unfortunately, precludes the determination of the relative phase of the dipoles to the field which excites them. Nevertheless, the analysis can still be used to quantify the amplitude of both the electric and magnetic polarizabilities of the hole, as we show below.

We first examine the field patterns of the scattered SPP wave. Figures 3(a) and 3(b) show the two calculated in-plane electric field components of the scattered field, corresponding to the configuration considered in Fig. 2. These radiation patterns show that even a process as elementary as a single circular hole scattering SPPs can result in a nontrivial distribution of surface electromagnetic fields. We see the asymmetry of the scattered field profile from the angular distributions of these radiation patterns, which we present in Figs. 3(c) and 3(d). Here we also show the contributions of the electric and magnetic dipoles to the overall scattered field. As expected, each individual contribution is symmetric, along both the $x$ and $y$ axes. However, symmetry with respect to the $y$ axis is broken for the total scattered field, resulting in a greater amount of light being scattered in the forward (with respect to the direction of propagation of the incident SPPs) direction. That is, in the forward direction the fields radiated by $p_{z}$ and
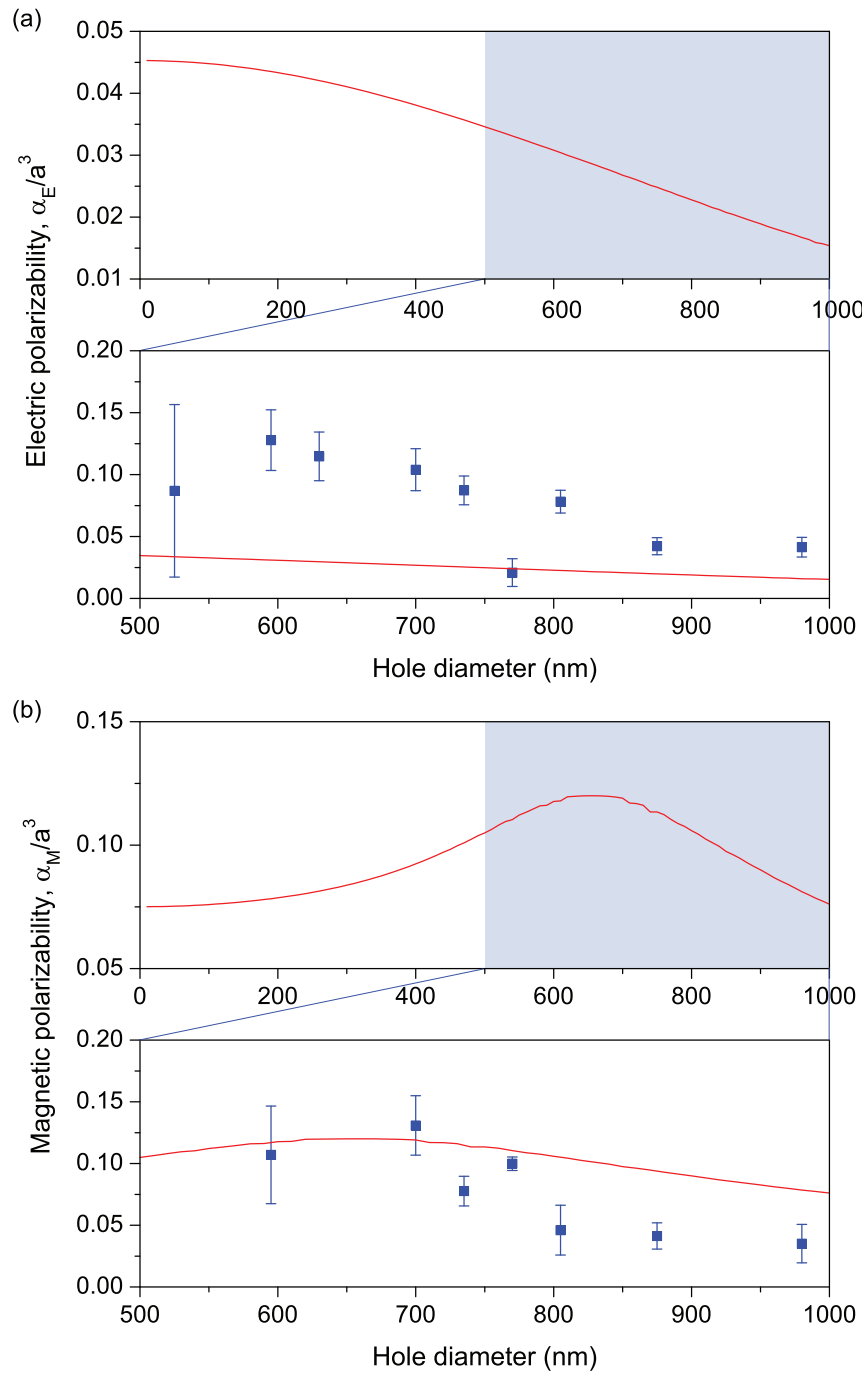

FIG. 4. (Color online) Electric (a) and magnetic (b) polarizabilities of individual holes as a function of diameter. The top panel of each part shows the calculated polarizabilities, while the bottom panel presents both the measured (points) and calculated (curves) values.

$m_{y}$ are more in phase, while they are more out of phase in the backward direction. The net result is that $60 \%$ of the field amplitude is radiated in the forward direction. Note that the directionality is solely determined by the relative phases of the electric and magnetic dipoles that, in turn, are determined by the phases of the incident fields and the polarizabilities of the hole. That is, this high directionality demonstrates the potential that nanoscopic structures have for controlling the flow of light, since their polarizabilities are often determined by their geometry. We emphasize that the directionality of the scattered field is due to Kerker scattering, which relies on the interference between the magnetic and electric contributions, ${ }^{29}$ as recently demonstrated by controlled illumination of a slit. ${ }^{9}$ This is in stark contrast to another recent demonstration of directional SPP excitation of a slit where only electric contributions were considered. ${ }^{8}$ In this latter case, for the slit, the ratio of the intensity scattered in the forward direction to that scattered backwards is about $1.4: 1$, whereas we measure a ratio of about $2.5: 1$ for the hole. 
As we noted above, we can use our analysis to quantify both the electric and magnetic response of the holes. In our approach, where we take dipoles to be the source of the scattered field, the optical response of the hole is approximated by the electric and magnetic polarizabilities, $\alpha_{E}$ and $\alpha_{M}$. These relate the induced dipoles to the incident fields as

$$
p_{z}=\alpha_{E} E_{z, 0}^{\text {in }}, \quad m_{y}=\alpha_{M} H_{y, 0}^{\text {in }},
$$

and hence, these parameters describe how strongly the hole reacts to the incident light. Because our model allows us to determine both the incident SPP fields [Eq. (1)] and the dipole strengths [Eq. (2)], we can use Eq. (3) to determine the polarizabilities of the hole.

In Fig. 4 we show a comparison of the experimentally determined $\alpha_{E}$ and $\alpha_{M}$ with theoretical values ${ }^{30}$ as a function of the hole diameter, where both quantities have been normalized to the cube of the hole radius, $a$. We observe good qualitative agreement for both $\alpha_{E}$ and $\alpha_{M}$, and very good quantitative agreement for the latter. As we note above, the uncertainty in the fitting of the exact hole position both limits our ability to determine the exact phase of the polarizabilities and introduces the main source of error in our analysis. Consequently, the error bars in Fig. 4 are found with a statistical analysis of the polarizabilities found when the hole position is allowed to vary within a 300-nm-diameter circle around $\left(x_{0}, y_{0}\right){ }^{31}$ Also note that $p_{z}$ radiates isotropically on the gold surface, whereas $m_{y}$ predominately radiates in the forward and backward directions [Figs. 3(c) and 3(d)]. Consequently, the magnetic contribution to the scattered field strongly overlaps with the incident SPP fields. Hence, for smaller hole sizes $(<700 \mathrm{~nm})$, where the amplitude of the scattered field is smaller, or on the order of, the noise in our measurements, we can often only determine $\alpha_{E}$ and not $\alpha_{M}$. In this case, it is reasonable to expect that $\alpha_{E}$ might be overestimated, which indeed agrees with our observations for the smaller holes. The polarizabilities that we do find are on the same order as those for off-resonance split rings. ${ }^{32}$
In summary, this work provides the key ingredient necessary to understand, and completely model, the light-matter interactions of holes: a quantification of the electromagnetic response of the hole. This quantification has long been missing in earlier theoretical studies of the microscopic optical properties of holes ${ }^{5,6}$ and newer experimental works on surface plasmon resonances, ${ }^{20}$ scattering losses,${ }^{33}$ the different fields present, ${ }^{22}$ or the near- ${ }^{23}$ and far-field ${ }^{24}$ radiation patterns associated with subwavelength holes.

Here, we have used a combination of phase- and polarization-sensitive near-field microscopy and electromagnetic theory to unravel the electric and magnetic polarizabilities of single subwavelength holes in optically thick gold films. This has allowed us to quantify the complete electromagnetic response of holes to surface waves, and in particular to demonstrate that the magnetic contribution should not be neglected, even for nanoscale geometries where the magnetic permeability of each constituent is always near unity. In fact, we show that for holes the magnetic response is often stronger than the electric, and that the interplay between the two can result in scattered field patterns that are highly directional. In our case, we showed that 2.5 times as much energy is scattered in the forward direction by the hole. However, with a careful choice of excitation field and hole parameters, one should be able to direct the scattering in any desired direction. ${ }^{9,34}$ As such, this understanding provides a crucial step towards the intelligent design of nanoscopic systems for the control and manipulation of the flow of light.

The authors thank M. P. van Exter for his insights and suggestions. This work is part of the research programme of the Foundation for Fundamental Research on Matter (FOM), which is part of the Netherlands Organisation for Scientific Research (NWO). This work is supported by NanoNextNL of the Dutch ministry EL\&I and 130 partners, and by the EU FET project "SPANGL4Q." F.J.G.deA. acknowledges support from the Spanish MEC (MAT2010-14885). *rotenberg@amolf.nl

†Present address: Zernike Institute for Advanced Materials, University of Groningen, Nijenborgh 4, 9747 AG Groningen, The Netherlands.

${ }^{\ddagger}$ Present address: Center for Solid State Physics and New Materials, Institute of Physics in Belgrade, Pregrevica 118, 11080 Belgrade, Serbia.

${ }^{1}$ C. J. Bouwkamp, Rep. Prog. Phys. 17, 35 (1954).

${ }^{2}$ V. M. Shalaev, Nat. Photonics 1, 41 (2007).

${ }^{3}$ D. R. Smith, J. B. Pendry, and M. C. K. Wiltshire, Science 305, 788 (2004).

${ }^{4}$ C. Genet and T. W. Ebbesen, Nature (London) 445, 39 (2007).

${ }^{5}$ H. Liu and P. Lalanne, Nature (London) 452, 728 (2008).

${ }^{6}$ F. J. García de Abajo, Rev. Mod. Phys. 79, 1267 (2007).

${ }^{7}$ A. G. Curto, G. Volpe, T. H. Taminiau, M. P. Kreuzer, R. Quidant, and N. F. van Hulst, Science 329, 930 (2010).

${ }^{8}$ F. J. Rodríguez-Fortuño, G. Marino, P. Ginzburg, D. O'Connor, A. Martínez, G. A. Wurtz, and A. V. Zayats, Science 340, 328 (2013).
${ }^{9}$ S.-Y. Lee, I.-M. Lee, J. Park, S. Oh, W. Lee, K.-Y. Kim, and B. Lee, Phys. Rev. Lett. 108, 213907 (2012).

${ }^{10}$ I. Sersic, M. Frimmer, E. Verhagen, and A. F. Koenderink, Phys. Rev. Lett. 103, 213902 (2009).

${ }^{11}$ M. Burresi, D. van Oosten, T. Kampfrath, H. Schoenmaker, R. Heideman, A. Leinse, and L. Kuipers, Science 326, 550 (2009).

${ }^{12}$ R. L. Olmon, M. Rang, P. M. Krenz, B. A. Lail, L. V. Saraf, G. D. Boreman, and M. B. Raschke, Phys. Rev. Lett. 105, 167403 (2010).

${ }^{13}$ H. W. Kihm, S. M. Koo, Q. H. Kim, K. Bao, J. E. Kihm, W. S. Bak, S. H. Eah, C. Lienau, H. Kim, P. Nordlander, N. J. Halas, N. K. Park, and D.-S. Kim, Nat. Commun. 2, 451 (2011).

${ }^{14}$ T. H. Taminiau, S. Karaveli, N. F. van Hulst, and R. Zia, Nat. Commun. 3, 979 (2012).

${ }^{15}$ I. Staude, A. E. Miroshnichenko, M. Decker, N. T. Fofang, S. Liu,

E. Gonzales, J. Dominguez, T. S. Luk, D. N. Neshev, I. Brener, and Y. Kivshar, ACS Nano 7, 7824 (2013)

${ }^{16}$ S. Person, M. Jain, Z. Lapin, J. J. Sáenz, G. Wicks, and L. Novotny, Nano Lett. 13, 1806 (2013).

${ }^{17}$ Y. H. Fu, A. I. Kuznetsov, A. E. Miroshnichenko, Y. F. Yu, and B. Luk'yanchuk, Nat. Commun. 4, 1527 (2013). 
${ }^{18}$ C. S. Levin, C. Hofmann, T. A. Ali, A. T. Kelly, E. Morosan, P. Nordlander, K. H. Whitmire, and N. J. Halas, ACS Nano 3, 1379 (2009).

${ }^{19}$ J. A. Fan, C. Wu, K. Bao, J. Bao, R. Bardhan, N. J. Halas, V. N. Manoharan, P. Nordlander, G. Shvets, and F. Capasso, Science 328, 1135 (2010).

${ }^{20}$ J. Prikulis, P. Hanarp, L. Olofsson, D. Sutherland, and M. Käll, Nano Lett. 4, 1003 (2004).

${ }^{21}$ F. de León-Pérez, G. Brucoli, F. J. García-Vidal, and L. MartínMoreno, New J. Phys. 10, 105017 (2008).

${ }^{22}$ F. van Beijnum, C. Rétif, C. B. Smiet, H. Liu, P. Lalanne, and M. P. van Exter, Nature (London) 492, 411 (2012).

${ }^{23}$ N. Rotenberg, M. Spasenović, T. L. Krijger, B. le Feber, F. J. García de Abajo, and L. Kuipers, Phys. Rev. Lett. 108, 127402 (2012).

${ }^{24}$ J.-M. Yi, A. Cuche, F. de León-Pérez, A. Degiron, E. Laux, E. Devaux, C. Genet, J. Alegret, L. Martín-Moreno, and T. W. Ebbesen, Phys. Rev. Lett. 109, 023901 (2012).

${ }^{25}$ F. López-Tejeira, S. G. Rodrigo, L. Martín-Moreno, F. J. GarcíaVidal, E. Devaux, T. W. Ebbesen, J. R. Krenn, I. P. Radko, S. I. Bozhevolnyi, M. U. González, J. C. Weeber, and A. Dereux, Nat. Phys. 3, 324 (2007).
${ }^{26}$ M. Sandtke, R. J. P. Engelen, H. Schoenmaker, I. Attema, H. Dekker, I. Cerjak, J. P. Korterik, F. B. Segerink, and L. Kuipers, Rev. Sci. Instrum. 79, 013704 (2008).

${ }^{27}$ P. Lalanne and J. P. Hugonin, Nat. Phys. 2, 551 (2006).

${ }^{28}$ P. B. Johnson and R. W. Christy, Phys. Rev. B 6, 4370 (1972).

${ }^{29}$ M. Kerker, D.-S. Wang, and C. L. Giles, J. Opt. Soc. Am. 73, 765 (1983).

${ }^{30}$ Details for these calculations, which are performed for holes in a perfectly conducting metal film, can be found in the Supplemental Material of Ref. 23.

${ }^{31}$ This circle size represents our resolution, which is of the order of the aperture size of our near-field tip. The error bars denote the standard deviation of the polarizabilities found from the fits for 36 equally spaced hole positions within this region.

${ }^{32}$ F. B. Arango and A. F. Koenderink, New J. Phys. 15, 073023 (2013).

${ }^{33}$ F. van Beijnum, J. Sirre, C. Rétif, and M. P. van Exter, Phys. Rev. B 85, 035437 (2012).

${ }^{34}$ J. P. Balthasar Mueller and F. Capasso, Phys. Rev. B 88, 121410(R) (2013). 